\title{
Wnt3a expression is associated with MMP-9 expression in primary tumor and metastatic site in recurrent or stage IV colorectal cancer
}

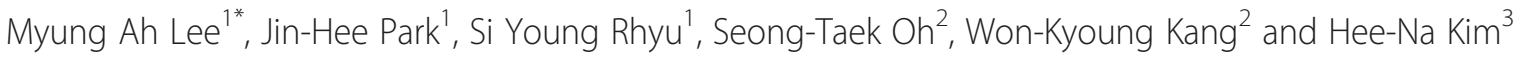

\begin{abstract}
Background: The wnt/ $\beta$-catenin signaling pathway is known to affect in cancer oncogenesis and progression by interacting with the tumor microenvironment. However, the roles of wnt3a and wnt5a in colorectal cancer (CRC) have not been thoroughly studied. In the present study, we investigated the expression of wht protein and the concordance rate in primary tumor and metastatic sites in CRC. To determine the relationship of wnt proteins with invasion related protein, we also analyzed the association between wht protein expression and the expression of matrix metalloproteinase-9 (MMP-9) and vascular endothelial growth factor receptor-2 (VEGFR-2).
\end{abstract}

Methods: Tumor tissue was obtained from eighty-three paraffin- embedded blocks which were using resected tissue from both the primary tumor and metastatic sites for each patient. We performed immunohistochemical staining for wnt3a, wnt5a, $\beta$-catenin, MMP-9 and VEGFR-2.

Results: Wnt3a, wnt5a, $\beta$-catenin, and MMP-9 expression was high; the proteins were found in over $50 \%$ of the primary tumors, but the prevalence was lower in tissue from metastatic sites. The concordance rates between the primary tumor and metastatic site were $76.2 \%$ for wnt5a and $79.4 \%$ for wnt3a and $\beta$-catenin, but VEGFR- 2 was expressed in $67.4 \%$ of the metastatic sites even when not found in the primary tumor. Wnt3a expression in primary tumors was significantly associated with lymph node involvement $(p=0.038)$ and MMP-9 expression in the primary tumor $(p=0.0387)$, mesenchyme adjacent to tumor $(p=0.022)$ and metastatic site $(p=0.004)$. There was no other relationship in the expression of these proteins. Vascular invasion in primary tumor tissue may be a potential prognostic marker for liver metastasis, but no significant association was observed among the wnt protein, MMP-9, and VEGFR-2 for peritoneal seeding. In survival analysis, $\beta$-catenin expression was significantly correlated with overall survival $(p=0.05)$.

Conclusions: Wnt3a and wnt5a expression had a concordance rate higher than $60 \%$ with a high concordance rate between the primary tumor and metastatic site. Wnt3a expression is associated with the expression of MMP-9 in primary tumor tissue adjacent mesenchymal tissue, and at the metastatic site. As a prognostic marker, only $\beta$-catenin expression showed significant relation with survival outcome.

Keywords: Wnt3a, Wnt5a, MMP-9, VEGFR-2, $\beta$-catenin, Colorectal cancer

\section{Background}

Colorectal cancer (CRC) is the $3^{\text {rd }}$ most common cancer in Korea and is becoming more common in Asia and Western countries [1]. As new anti-cancer agents and new technology for local treatment have been developed, the survival rates of patients with CRC have been

\footnotetext{
* Correspondence: angelamd@catholic.ac.kr

'Division of Medical Oncology, Department of Internal Medicine, Cancer Research Institute, College of Medicine, The Catholic University of Korea, Seoul St. Mary's Hospital, 222 Banpo-daero, Seocho-gu, 137-701 Seoul, Korea Full list of author information is available at the end of the article
}

increasing, even for patients with stage IV cancer. However, distant organ metastasis eventually develops in stage IV cancer, and leads to fatal organ failure. To improve survival outcomes, it is important to control cancer invasion and metastasis, but the mechanisms by which CRC becomes metastasis are not yet known.

The wnt/ $\beta$-catenin pathway is known to play an important role in maintaining cell homeostasis in normal cells and in embryologic cell development. In CRC, mutation in APC or $\beta$-catenin, component of the wnt signaling 
pathway, are well-known oncogenic factors in familial and some sporadic CRC cases. Recently, many researchers have suggested that the wnt signaling pathway is also involved in controlling cancer cell invasion and metastasis by interacting with the tumor microenvironment or other signal pathways [2-4]. However, most studies investigating the effects of the wnt pathway on CRC have focused on $\beta$-catenin, and the role of wnt3a (the initiator of wnt/ $\beta$-catenin pathway) is not well understood.

The non-canonical pathway via wnt5a is another of the identified wnt signaling pathways. The non-canonical pathway plays a role in embryonic cell motility, but the role of the non-canonical pathway in cancer is unknown. Katoh has suggested that the non-canonical pathway is involved in tumor cell invasion and metastasis [5], but studies investigating the role of wnt5a expression in cancer have been limited and controversial. Several authors have reported that the wnt5a expression is associated with higher grade, poor differentiation or poor clinical outcomes, but others have suggested that wnt5a expression antagonizes the wnt $/ \beta$-catenin pathway and inhibits oncogenesis [6]. Dejimek et al. suggested that wnt5a expression in stage II colon cancer is associated with good prognosis, and another study reported that wnt5a methylation is associated with microsatellite instability and BRAF mutation $[7,8]$. Considering these data, the exact role of wnt5a in cancer is unclear.

MMP-9 and VEGF expression are commonly studied in cancer research, and their expression is associated with invasion and metastasis. One study reported that in vitro inhibition of DKK-1 signaling inhibits the MMP-9 expression [9], but MMP-9 expression decreased after wnt3a treatment in another study [10]. Many studies investigating the role of MMP-9 in CRC have been conducted using cell lines or animal models. Few trials have reported that MMP-9 expression can be used as a prognostic factor for disease recurrence in human tissue [11]. Thus, the association between the wnt signaling pathway and MMP9 and VEGFR-2 expression in CRC remains unclear.

Previous experiments investigating the role of the wnt proteins in metastasis have been conducted at the primary site of stages I-III CRC because diseased tissue can be easily obtained through surgery. Studies focusing on the relationships between the primary tissue and tissue from the metastatic site have rarely been reported. Recently, resection of metastatic lesions has become a treatment option for patients with stage IV CRC. In the present study, we evaluated the expression of wnt3a, wnt5a, MMP-9, and VEGFR-2 in human tissue from primary and metastatic sites of stage IV advanced CRC patients to identify associations between these proteins. We also analyzed the concordance between the primary and metastatic lesions, and aimed to identify the potential prognostic markers of survival outcome.

\section{Methods}

\section{Patients}

This study included eighty-three patients with colon or rectal cancer who had resection for both a primary mass and metastatic lesions resected in a single procedure at Seoul St. Mary's Hospital between January 2000 and December 2006. All patients had colorectal cancer with organ metastasis at the initial diagnosis. Clinical records and pathological reports were reviewed retrospectively. This study was approved by the institutional review board of Seoul St. Mary's hospital (KC10SIMI0621).

\section{Tissue microarray (TMA) and immunohistochemical staining} Core biopsies with $3.0 \mathrm{~mm}$ diameter were taken from representative areas of tumor tissue. Each patient had biopsy samples taken from the primary tumor mass and its adjacent mesenchyme as well as the metastatic site and its adjacent mesenchyme. Tissue cores from each specimen were assembled on a recipient paraffin block with a precision instrument (Micro Digital Co. Korea), following previously established methods [12].

We performed immunohistochemical staining on $5 \mu \mathrm{m}$ sections of the TMA blocks. Each paraffin section was deparaffinized for 1 hour at $60^{\circ} \mathrm{C}$ in xylene and rehydrated in serial graded ethanol before being stored overnight in citrate buffer ( $0.01 \mathrm{M}, \mathrm{pH} 6.0)$ at $75^{\circ} \mathrm{C}$ for antigen retrieval. Endogenous peroxidase activity was blocked with $0.3 \%$ hydrogen peroxide in methanol. Sections were incubated for 1 hour at room temperature with the following primary antibodies at the specified dilutions: Wnt3a (Abcam, Cambridge, UK) diluted 1:100, Wnt5a (Abcam, Cambridge, UK) diluted 1:50, $\beta$-catenin (Abcam, Cambridge, UK) diluted 1:100, MMP-9 (Cell Signaling, Danvers, MA, USA) diluted 1:100, and VEGFR-2 (Cell Signaling, Danvers, MA, USA) diluted 1:200. Immunohistochemical staining was performed using the rabbit or mouse DAKO ChemMate TM EnVision TM system and a Peroxidase/ DAB kit (DAKO). Sections were then counterstained with Mayer hematoxylin and dehydrated, cleared and mounted.

The results were interpreted by two independent pathologists who were blinded to the specific diagnosis and prognosis for each case. The staining intensity was scored on a three-tiered scale: score $0=$ less than $10 \%$ of cells positive; $1=10-49 \%$ positive; and $2=$ more than $50 \%$ of cells positive. The criterion for positive staining was more than $1+$ of tumor cells that showed distinct nuclear or cytoplasmic staining (Figure 1).

\section{Statistical analysis}

Continuous and categorical variables were compared using the Student's $t$ test and chi-square test. All statistical analyses were performed using SPSS (version 13.0) and $\mathrm{p}$ value under 0.05 was considered statistically significant. 


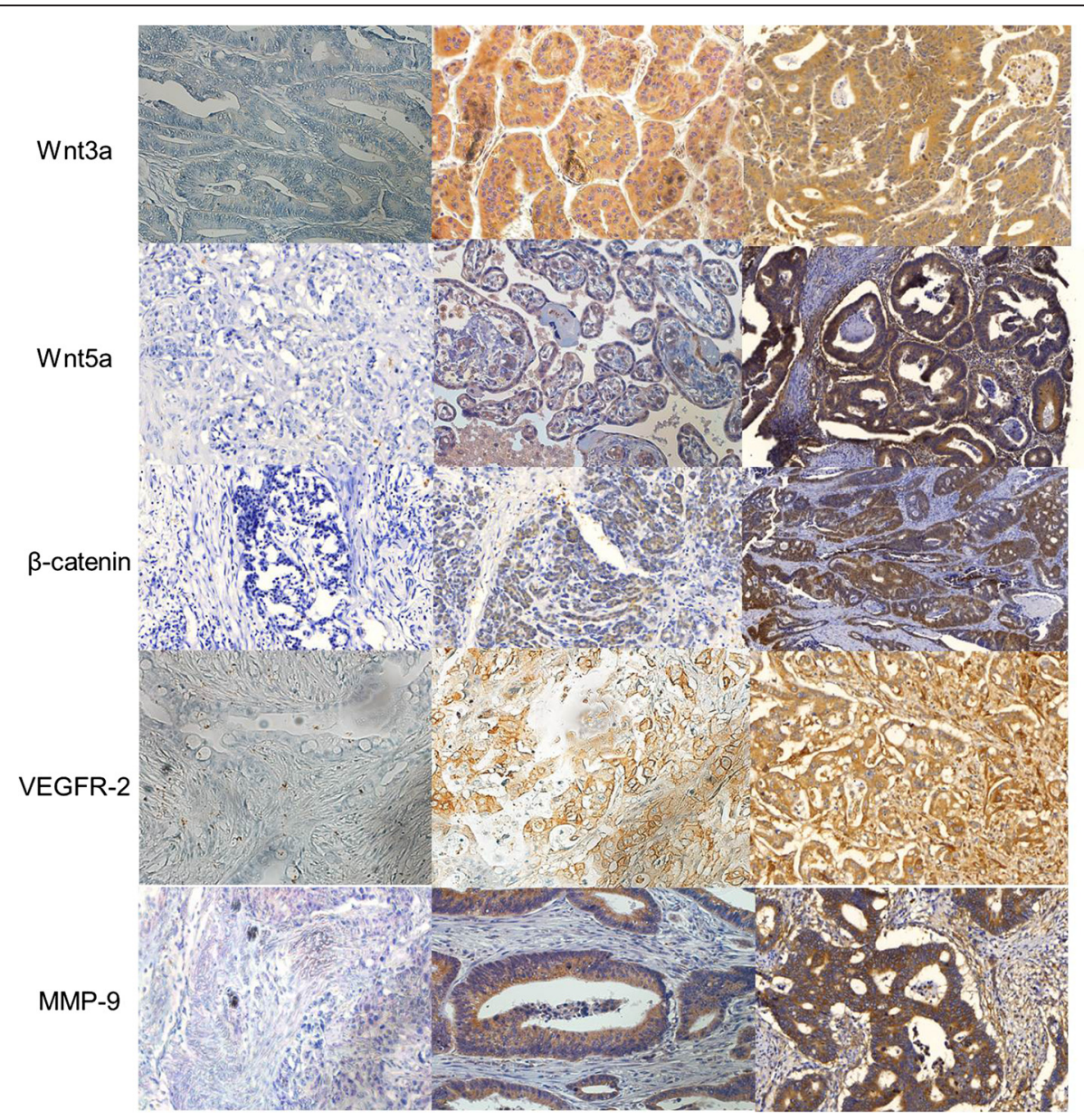

Figure 1 Immunohistochemical staining for the wnt3a, wnt5a, $\beta$-catenin, MMP-9, and VEGFR-2 (magnification $\times 200$ ).

\section{Results}

Patient characteristics and protein expression

Of the 83 patients, 46 were male and the median age was 60 years. Liver metastasis was the most common $(57.8 \%)$, followed by peritoneum metastasis (26.5\%). Thirteen patients had no lymph node involvement even with stage IV disease, and two patients had T2 disease. The patient characteristics are summarized in Table 1.

Wnt3a, wnt5a, MMP-9 and $\beta$-catenin were expressed in more than $50 \%$ of the primary tumors, but VEGFR-2 was not. These protein expression levels were slightly decreased in the tissue taken from the metastatic sites (Table 2). We analyzed the concordance rate of the protein expression between the primary tumor and metastatic site. The concordance rates of wnt3a, wnt5a and $\beta$-catenin expression were high; all of the rates were in the range of $76.2 \%$ to $79.4 \%$, and MMP-9 expression had a $68.3 \%$ concordance rate. However, VEGFR-2 was expressed in $67.4 \%$ of the metastatic sites when there was no expression in the primary tumors, with only a $40.0 \%$ concordance rate between primary tumor and metastatic sites.
Wnt3a expression in primary tumor correlated with lymph node involvement and MMP-P expression

We analyzed the association between wnt expression in the primary tumor and the clinicopathologic findings, including the $\mathrm{T} \& \mathrm{~N}$ stage. Wnt3a expression in the primary tumor was significantly correlated with lymph node involvement $(\mathrm{p}=0.038)$ and MMP-9 expression in primary, adjacent mesenchyme and metastatic sites ( $\mathrm{p}=0.038,0.022$ and 0.004 , respectively). There was no association between wnt5a expression and other findings, but wnt5a expression did show a correlation tendency with lymph node and lymphatic invasion. This result is summarized in Table 3.

Analysis of liver or peritoneal metastasis, wnt expression, MMP expression, and VEGFR-2 expression in the primary tumor did not show any associations but venous invasion was associated with liver metastasis $(\mathrm{p}=0.047)$. We also performed immunohistochemical staining of the adjacent mesenchymal tissue, but there was no association between wnt3a, wnt5a, MMP, or VEGFR-2 expression and liver or peritoneal metastasis (Table 4). 
Table 1 Patients' characteristics

\begin{tabular}{ccc}
\hline & & Number \\
\hline Age & Years & $60(27-78)$ \\
Sex & M : F & $46: 37$ \\
Location & Colon & $51(61.4 \%)$ \\
$T$ & Rectum & $32(38.6 \%)$ \\
& 2 & $2(2.4 \%)$ \\
$N$ & 3 & $24(28.9 \%)$ \\
& 4 & $57(68.7 \%)$ \\
& 0 & $13(15.7 \%)$ \\
Metastasis & 1 & $26(31.3 \%)$ \\
& 2 & $44(53.0 \%)$ \\
& Liver & $48(57.8 \%)$ \\
& Peritoneum & $22(26.5 \%)$ \\
& Lung & $4(4.8 \%)$ \\
& Ovary & $3(3.6 \%)$ \\
& Others & $6(7.2 \%)$ \\
\hline
\end{tabular}

In survival analysis, patients with positive $\beta$-catenin staining in primary tumors showed significantly poorer survival outcomes than those with no staining (18.4 months vs. 42.9 months, respectively, $\mathrm{p}=0.05$, Figure 2). There were no other prognostic factors for survival.

\section{Discussion}

The wnt/ $\beta$-catenin signaling pathway is an emerging target for cancer research; studies indication the pathway may play a role in cancer invasion and progression by interacting with the tumor microenvironment and oncogenesis [13]. Wnt/ $\beta$-catenin has been widely studied as a prognostic factor for $\mathrm{CRC}$, and it may also be involved in the mechanism of cancer invasion $[14,15]$. However, research has focused on aberrant nuclear $\beta$-catenin rather than wnt3a, despite the role of wnt3a as a major initiating factor in the wnt $/ \beta$-catenin pathway. In the present study, we analyzed wnt3a and wnt5a to determine their roles in cancer progression. We selected the patients who had had both their primary tumor and the metastatic sites resected to identify differences in the protein expression between the primary and the metastatic sites. Expression of wnt3a was very high in tissues from both primary tumors and metastatic sites but was higher at the primary site with a concordance rate higher than $70 \%$. Wnt expression at the metastatic site was rare if the primary tumor tested
Table 3 The association of wnt expression in primary tumor and other pathologic findings

\begin{tabular}{|c|c|c|c|c|c|}
\hline & & \multicolumn{2}{|c|}{ Wnt3a } & \multicolumn{2}{|c|}{ Wnt5a } \\
\hline & & No. & $\mathbf{p}$ & No. & $\mathbf{p}$ \\
\hline \multirow[t]{3}{*}{$\mathrm{T}$} & 2 & 1 & & 0 & \\
\hline & 3 & 13 & 0.626 & 17 & 0.092 \\
\hline & 4 & 37 & & 41 & \\
\hline \multirow[t]{3}{*}{$\mathrm{N}$} & 0 & 8 & & 9 & \\
\hline & 1 & 21 & 0.038 & 19 & 0.910 \\
\hline & 2 & 22 & & 30 & \\
\hline \multirow[t]{3}{*}{ Grade } & Well & 6 & & 6 & \\
\hline & Moderate & 37 & 0.268 & 45 & 0.945 \\
\hline & Poorly & 8 & & 7 & \\
\hline Lymphatic invasion & $(+)$ & 46 & 0.482 & 54 & 0.088 \\
\hline Venous invasion & $(+)$ & 16 & 0.594 & 20 & 0.249 \\
\hline Perineural invasion & $(+)$ & 34 & 0.060 & 37 & 0.136 \\
\hline \multirow[t]{3}{*}{ Primary tumor } & $\beta$-catenin & 46 & 0.063 & 52 & 0.054 \\
\hline & MMP-9 & 34 & 0.004 & 33 & 0.306 \\
\hline & VEGFR-2 & 21 & 0.166 & 24 & 0.102 \\
\hline Adjacent & $\beta$-catenin & 39 & 0.299 & 37 & 0.289 \\
\hline \multirow[t]{2}{*}{ Mesemchyme } & MMP-9 & 32 & 0.022 & 32 & 0.359 \\
\hline & VEGFR-2 & 15 & 0.205 & 17 & 0.158 \\
\hline \multirow[t]{3}{*}{ Metastatic site } & $\beta$-catenin & 29 & 0.382 & 32 & 0.598 \\
\hline & MMP-9 & 23 & 0.031 & 20 & 0.511 \\
\hline & VEGFR-2 & 16 & 0.396 & 15 & 0.364 \\
\hline
\end{tabular}

negative for both wnt3a and wnt5a. This result suggests that wnt could be expressed initially when CRC develops, not newly emerged as the cancer progresses. This result is consistent with the previous studies on the oncogenic role of the wnt signaling pathway in various cancers $[16,17]$. Recently, Boutros reported that sustained wnt activity through wnt3a and Evi/Wls/GPR177 can be important for the proliferation in colon cancer cell, independently from APC or $\beta$-catenin mutation [18]. It suggests that the upper stream factor of wnt signaling pathway may play an important role in the cancer progression.

It is well understood that MMP-9 overexpression is a key factor in degradation of the extracellular matrix, an essential step in tumor invasion and metastasis; this role has been observed in human tissue and cell line studies of CRC $[19,20]$. In the present study, wnt3a expression was significantly correlated with MMP-9 expression in the

Table 2 Immunohistochemical staining for primary tumors and metastatic site

\begin{tabular}{lccccc}
\hline & Wnt3a & Wnt5a & $\beta$-catenin & MMP-9 & VEGFR-2 \\
\hline Primary tumor & $51(61.4 \%)$ & $58(69.9 \%)$ & $70(84.3 \%)$ & $45(54.2 \%)$ & $30(36.1 \%)$ \\
Metastatic site & $38(45.8 \%)$ & $39(47.0 \%)$ & $44(53.0 \%)$ & $28(33.7 \%)$ & $22(26.5 \%)$ \\
Concordance rate & $79.4 \%$ & $76.2 \%$ & $79.4 \%$ & $68.3 \%$ & $40.0 \%$ \\
\hline
\end{tabular}


Table 4 The association between the protein expression and liver or peritoneal seeding

\begin{tabular}{|c|c|c|c|c|c|}
\hline & \multicolumn{2}{|c|}{ Liver } & \multicolumn{2}{|c|}{ Peritoneum } \\
\hline & & No. & $\mathrm{p}$ & No. & $\mathrm{p}$ \\
\hline \multirow[t]{3}{*}{ T } & 2 & 1 & & 0 & \\
\hline & 3 & 18 & 0.130 & 4 & 0.264 \\
\hline & 4 & 29 & & 18 & \\
\hline \multirow[t]{3}{*}{$\mathrm{N}$} & 0 & 6 & & 2 & \\
\hline & 1 & 17 & 0.508 & 5 & 0.243 \\
\hline & 2 & 25 & & 15 & \\
\hline \multirow[t]{3}{*}{ Grade } & Well & 4 & & 3 & \\
\hline & Moderate & 39 & 0.749 & 17 & 0.419 \\
\hline & Poorly & 5 & & 2 & \\
\hline Lymphatic invasion & $(+)$ & 44 & 0.304 & 20 & 0.556 \\
\hline Venous invasion & $(+)$ & 19 & 0.047 & 5 & 0.230 \\
\hline Perineural invasion & $(+)$ & 27 & 0.353 & 15 & 0.223 \\
\hline \multirow[t]{5}{*}{ Primary tumor } & Wnt3a & 30 & 0.498 & 13 & 0.493 \\
\hline & Wnt5a & 34 & 0.506 & 12 & 0.062 \\
\hline & $\beta$-catenin & 39 & 0.277 & 18 & 0.470 \\
\hline & MMP-9 & 26 & 0.584 & 10 & 0.238 \\
\hline & VEGFR-2 & 15 & 0.196 & 9 & 0.385 \\
\hline \multirow[t]{5}{*}{ Adjacent mesenchyme } & Wnt3a & 18 & 0.498 & 11 & 0.151 \\
\hline & Wnt5a & 29 & 0.298 & 15 & 0.412 \\
\hline & $\beta$-catenin & 36 & 0.453 & 15 & 0.347 \\
\hline & MMP-9 & 25 & 0.510 & 12 & 0.533 \\
\hline & VEGFR-2 & 13 & 0.431 & 5 & 0.494 \\
\hline
\end{tabular}

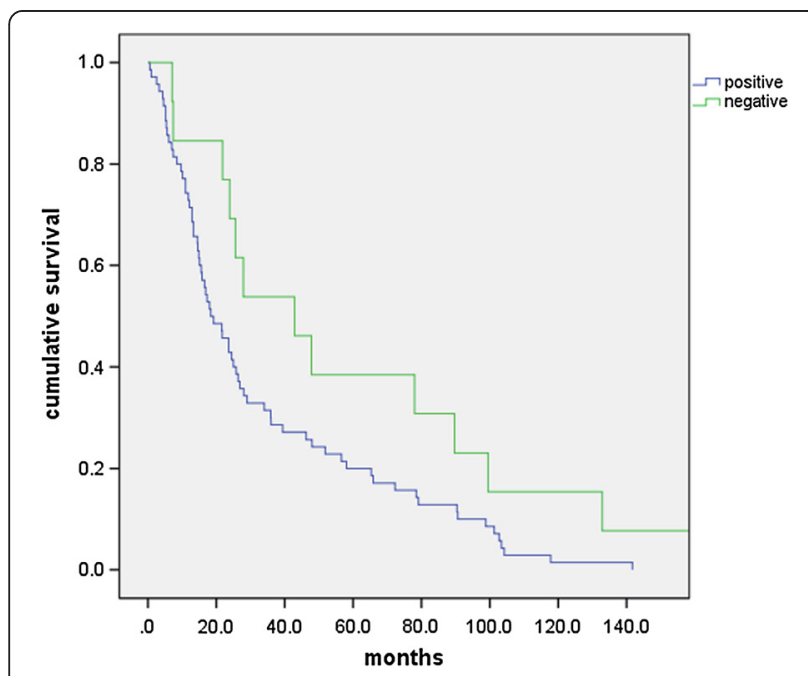

Figure 2 Overall survival according to $\beta$-catenin expression.

$\beta$-catenin-expressing group in primary tumor showed poorer survival outcome than non-expressing group (18.4 vs. 42.9 months, $p=0.05$ ). primary tumor, mesenchyme and metastatic site. In previous study, inhibition of the wnt/ $\beta$-catenin pathway decreased the level of MMP-9 mRNA in embryonic neural stem cells [9]. These data suggest that the wnt $/ \beta$-catenin signaling pathway may have an effect on MMP-9 expression, with a role in cancer invasion and metastasis. However, in one mouse study, wnt3a stimulation was shown to inhibit MMP-2 and MMP-9 expression in mesenchymal stem cells; the investigator suggested that regulation of wnt3a could be different in mice and human [13]. Recently, some studies have reported that inhibition of $\beta$-catenin by some agents can also inhibit MMP- 2 or MMP-9 expression $[21,22]$. The previous data suggest that the wnt/ $\beta$-catenin pathway may play a role in cancer invasion and metastasis through MMP-9 expression.

VEGFR is also a topic of interest in cancer proliferation and metastasis research. Many studies have associated the wnt/ $\beta$-catenin signaling pathway with VEGFR activity $[23,24]$. In this study, however, wnt expression was not correlated with VEGFR-2 expression. In addition, VEGFR2 expression was relatively low in our study, particularly at the metastatic site. In other experiments, VEGFR-1 expression was regulated independent of the wnt $/ \beta$-catenin pathway, and VEGFR-2 did not show any significant association with lymph node or lymphovascular invasion $[25,26]$. Based on these data, the mechanism by which VEGFR is involved in metastasis should be explored independently of the wnt signaling pathway.

Wnt5a is a key initiating factor in the non-canonical pathway, but its role in cancer is not known. Kato has suggested that the non-canonical pathway may be involved in cancer cell invasion [5]. In previous study, wnt5a expression showed aggressive behavior in breast or gastric cancer $[27,28]$. However, wnt5a has also been associated with a good prognosis or tumor suppression by inhibiting the wnt $/ \beta$-catenin pathway in CRC $[7,29]$. In our study, wnt5a showed no correlation with pathologic findings or invasion related protein expression, but showed higher expression in the primary and metastatic tumor sites. The data do not show an antagonistic relationship between wn3a and wnt5a in the present study. To determine the role of wnt5a in CRC, further analysis of other signaling pathways is warranted.

Theoretically, wnt3a expression is directly associated with $\beta$-catenin expression. However, previous studies have reported that $\beta$-catenin can be independently, aberrantly expressed without altering wnt3a in CRC $[14,15]$ and could not be differentiated from the $\beta$-catenin that is activated by wnt3a. This is the reason $\beta$-catenin was higher than wnt3a expression in our study. In survival analysis of our study, $\beta$ catenin expression was significantly correlated with poor survival outcome, independently of wnt3a expression. It has previously been shown that the $\beta$-catenin expression can be independent prognostic marker for CRC patients. 
As a prognostic factor for overall survival, $\beta$-catenin expression was significantly correlated only with the survival outcome. Known prognostic factors, such as lymph node involvement or lymphovascular invasion, did not show any significance in our survival analysis. We analyzed the stage IV patients with metastasis in the present study; these factors could have less of effect on the survival status in stage IV patients than in stage II or III CRC patients.

There is a limitation in our study. We could not determine whether the wnt and MMP-9 expression levels are prognostic or predictive factors because we performed the present study in stage IV CRC patients. According to the objective of this study, we enrolled the patients who underwent surgery for primary and metastatic sites; thus, patients with early stages of CRC were not included. Therefore, a comparative study would be required to determine whether wnt and MMP-9 expression levels are prognostic factors for the recurrence of distant metastasis.

In summary, wnt3a and wnt5a expression is high in primary and metastatic tumors in CRC with a high concordance rate. The wnt3a expression is highly correlated with MMP-9 expression, but not with VEGFR-2, and we did not determine the role of wnt5a. To investigate the mechanism of invasion and metastasis, further studies of the wnt/ $\beta$-catenin pathway and MMP-9 should be performed, and another approach for evaluating VEGFR or wnt5a should be explored.

\section{Conclusions}

Wnt3a and wnt5a are highly expressed in colorectal cancer both in primary and metastatic sites with a higher than $50 \%$ concordance rate. The wnt3a expression is significantly associated with MMP-9 expression, the metastasis related protein, but is not related with VEGFR-2 expression, and other metastatic related protein.

\section{Abbreviation}

CRC: Colorectal cancer; MMP-9: Matrix metalloprroteinase-9; TMA: Tissue microarray; VEGF: Vascular endothelial growth factor; VEGFR: Vascular endothelial growth factor receptor.

\section{Competing interests}

The authors declare that they have no competing interests.

\section{Authors' contributions \\ MAL suggested the idea and designed the all research process, took part in acquision of clinical data, analyzed \& interpreted of all the data, finally drafted \& revised the manuscript. JHP performed the experimental, analyzed the data and reviewed the manuscript. SYR was in charge of collecting all the clinical data, analyed and interpreted the data. STO \& WKK supplied all the tissue specimen, collected and interpreted data, and reviewed \& commented the manuscript. HNK interpreted the pathological findings, immunohistochemical staining, reviewed \& commented the manuscript. All authors read and approved the final manuscript.}

\section{Acknowledgements}

This research was supported by the financial support of the Catholic Medical Center Research Foundation made in the program year of 2010 and Seoul St. Mary's Clinical Medicine Research Program year of 2009-2011 through the Catholic University of Korea.

\section{Author details}

${ }^{1}$ Division of Medical Oncology, Department of Internal Medicine, Cancer Research Institute, College of Medicine, The Catholic University of Korea, Seoul St. Mary's Hospital, 222 Banpo-daero, Seocho-gu, 137-701 Seoul, Korea. ${ }^{2}$ Department of Surgery, Seoul St. Mary's Hospital, The Catholic University of Korea, Seoul, Korea. ${ }^{3}$ Department of Pathology, Seoul Clinical Laboratory Clinic, Seoul, Korea.

Received: 31 May 2013 Accepted: 13 February 2014

Published: 24 February 2014

\section{References}

1. Jung KW, Park SH, Won YJ, Kong YJ, Lee JY, Park EC, Lee JS: Prediction of cancer incidence and mortality in Korea. Cancer Res Treat 2011, 43(1):12-18. 2. Polarkis P: Wnt signaling and cancer. Genes Dev 2000, 14:1837-1851.

3. Saif MW, Chu E: Biology of colorectal cancer. Cancer J 2010, 16(3):196-201.

4. Huang D, Du X: Crosstalk between tumor cells and microenvironment via wnt pathway in colorectal cancer dissemination. J Gastroenterol 2008, 14(12):1823-1827.

5. Katoh M: WNT/PCP signaling pathway and human cancer (review). Oncol Repub 2005, 14(6):1583-1588.

6. McDonald SL, Silver A: The opposing roles of wnt-5a in cancer. $\mathrm{Br} J \mathrm{Can}$ 2009, 101:209-214.

7. Dejimek J, Dejimek A, Säfholm A, Sjölander A, Andersson T: Wnt-5a protein expression in primary dukes $B$ colon cancers identifies a subgroup of patients with good prognosis. Cancer Res 2005, 65(20):9142-9146.

8. Rawson JB, Mrkonjic M, Daftary D, Dicks E, Buchanan DD, Younghusband HB, Parfrey PS, Young JP, Pollett A, Green RC, Gallinger S, McLaughlin JR, Knight JA, Bapat B: Promoter methylation of Wnt5a is associated with microsatellite instability and BRAF V600E mutation in two large populations of colorectal cancer patients. Br J Cancer 2011, 104(12):1906-1912.

9. Ingraham CA, Park GC, Makarenkova HP, Crossin K: Matrix Metalloproteinase (MMP)-9 induced by wnt signaling increases the proliferation and migration of embryonic neural stem cells at low $\mathrm{O} 2$ levels. J Bio Chem 2011, 286(20):17649-17657.

10. Karow M, Popp T, Egea V, Ries C, Jochum M, Neth P: Wnt signaling in muse mesenchymal stem cells: impact on proliferation, invasion and MMP expression. J Cell Mol Med 2009, 13(88):2506-2520.

11. Bendardaf $R$, Buhmeida $A$, Hilska $M$, Laato $M$, Syrjänen $S$, Syrjänen $K$, Collan $Y$, Pyrhönen S: MMP-9 (gelatinase B) expression is associated with disease-free survival and disease-specific survival in colorectal cancer patients. Cancer Invest 2010, 28(1):38-43.

12. Lee MA, Park KS, Lee HJ, Jung JH, Kang JH, Hong YS, Lee KS, Kim DG, Kim SN: Survivin expression and its clinical significance in pancreatic cancer. BMC Cancer 2005, 5:127-132.

13. Neth $P$, Ciccarella M, Egea $V$, Hoelters J, Jochum M, Ries C: Wnt signaling regulates the invasion capacity of human mesenchymal stem cells. Stem Cells 2006, 24(8):1892-1903.

14. Elzagheid A, Buhmeida A, Korkeila E, Collan Y, Syrjanen K, Pyrhonen S: Nuclear beta-catenin expression as a prognostic factor in advanced colorectal carcinoma. World J Gastroenterol 2008, 14(24):3866-3871.

15. Suzuki H, Masuda N, Shimura T, Araki K, Kobayashi T, Tsutsumi S, Asao T, Kuwano $\mathrm{H}$ : Nuclear beta-catenin expression at the invasive front and in the vessels predicts liver metastasis in colorectal carcinoma. Anticancer Res 2008, 28(3B):1821-1830

16. Kahlil S, Tan GA, Giri DD, Zou XK, Howe LR: Activation status of Wnt/ $\beta$ catenin signaling in normal and neoplastic breast tissues: relationship to HER2/neu expression in human and mouse. PLos One 2012, 7(3):e33421.

17. Moyes LH, McEwan H, Radulescu S, Pawlikowski J, Lamm CG, Nixon C, Sansom OJ, Going JJ, Fullarton GM, Adams PD: Activation of Wnt signalling promotes development of dysplasia in Barrett's oesophagus. J Pathol 2012, 228(1):99-112.

18. Voloshanenko O, Erdmann G, Dubashi TD, Augustin I, Metzig M, Moffa G, Hundsrucker C, Ken G, Sandmann T, Anchang B, Demir K, Boehm C, Leible S, Ball CR, Glimm H, Spang R, Boutros M: Wnt secretion is required to maintain high levels of wnt activity in colon cancer cells. Nat Commun 2013, 4:2610.

19. Cheung LW, Leung PC, Wong AS: Gonadotropin-releasing hormone promotes ovarian cancer cell invasiveness through c-Jun NH2-terminal kinase-mediated activation of matrix metalloproteinase (MMP)-2 and MMP-9. Cancer Res 2006, 66(22):10902-10910. 
20. Roh SA, Choi EY, Cho DH, Jang SJ, Kim SY, Kim YS, Kim JC: Growth and invasion of sporadic colorectal adenocarcinomas in terms of genetic change. J Korean Med Sci 2010, 25:353-360.

21. Song KS, Li G, Kim JS, Jing K, Kim TD, Kim JP, Seo SB, Yoo JK, Park HD, Hwang BD, Lim K, Yoon WH: Protein-bound polysaccharide from Phellinus linteus inhibits tumor growth, invasion, and angiogenesis and alters Wnt/ $\beta$-catenin in SW480 human colon cancer cells. BMC Cancer 2011, 11:307.

22. Singh T, Katiyar SK: Honokiol Inhibits Non-Small Cell Lung Cancer Cell Migration by Targeting PGE2-Mediated Activation of $\beta$-Catenin Signaling. Plos One 2013, 8(3):e60749

23. Naik S, Dotharger RS, Marasa J, Lewis CL, Piwnica-Worms D: Vascular endothelial growth factor receptor-1 is synthetic lethal to aberrant beta catenin activation in colon cancer. Clin Cancer Res 2009, 15(24):7529-7537.

24. Zeitlin BD, Ellis LM, Nor JE: Inhibition of vascular endothelial growth factor receptor-1/wnt beta catenin cross talk leads to tumor cell death. Clin Cancer Res 2009, 15(24):7453-7455.

25. Yoshihara T, Takahashi-Yanaga F, Shiraishi F, Morimoto S, Watanabe Y, Hirata M, Hoka S, Sasaguri T: Anti-angiogenic effects of differentiation-inducing factor-1 involving VEGFR-2 expression inhibition independent of the Wnt/ß-catenin signaling pathway. Mol Cancer 2010, 16(9):245.

26. Kin JU, Bae BN, Kim HJ, Park KM: Prognostic significance of epidermal growth factor receptor and vascular endothelial growth factor receptor in colorectal adenocarcinoma. APMIS 2011, 119(7):449-459.

27. Kurayoshi M, Oue N, Yamamoto H, Kishida M, Inoue A, Asahara T, Yasui W, Kikuchi A: Expression of Wnt-5a is correlated with aggressiveness of gastric cancer by stimulating cell migration and invasion. Cancer Res 2006, 66(21):10439-10448.

28. Pukrop T, Klemm F, Hagemann T, Gradl D, Schulz M, Siemes S, Trümper L, Binder C: Wnt5a signaling is critical for macrophage-induced invasion of breast cancer cell lines. Proc Natl Acad Sci U S A 2006, 103(14):5454-5459.

29. Ying J, Li H, Yu J, Ng KM, Poon FF, Wong SC, Chan AT, Sung JJ, Tao Q: WNT5A exhibits tumor-suppressive activity through antagonizing the Wnt/beta-catenin signaling, and is frequently methylated in colorectal cancer. Clin Cancer Res 2008, 14(1):55-61.

doi:10.1186/1471-2407-14-125

Cite this article as: Lee et al.: Wnt3a expression is associated with MMP-9 expression in primary tumor and metastatic site in recurrent or stage IV colorectal cancer. BMC Cancer 2014 14:125.

\section{Submit your next manuscript to BioMed Central and take full advantage of:}

- Convenient online submission

- Thorough peer review

- No space constraints or color figure charges

- Immediate publication on acceptance

- Inclusion in PubMed, CAS, Scopus and Google Scholar

- Research which is freely available for redistribution 